\title{
In Vitro Oxygenation Injury to Slices Prepared from Ischemic Kidney in Rats
}

\author{
Kenji Asakura, Hanae Ikeda and Munekazu Gemba* \\ Division of Pharmacology, Osaka University of Pharmaceutical Sciences, Matsubara, Osaka 580, Japan
}

Received June 24, 1992 Accepted August 26, 1992

\begin{abstract}
When cortical slices prepared from rat kidneys made ischemic were incubated under a $100 \%$ oxygen atmosphere, lipid peroxidation increased and the ATP level decreased. Such oxygenation of the slices was accompanied by decreases in gluconeogenesis and the glutathione level, but an antioxidant, $N, N$ '-diphenyl-p-phenylenediamine, prevented the increase in lipid peroxidation without affecting decreases in ATP and glutathione levels, and gluconeogenesis. The results suggest that postischemic oxygenation of slices generates free radicals that cause the production of lipid peroxidation not associated with tissue injury.
\end{abstract}

Keywords: Ischemic kidney, Lipid peroxidation, Oxidative injury

Reperfusion injury is a pathophysiologic entity associated with the reintroduction of oxygen to ischemic tissues. Resumption of blood flow after arterial occlusion may generate oxygen radicals such as superoxide radical, hydrogen peroxide, and hydroxyl radical, which may be important in the genesis of postischemic tissue injury (1). Lipid peroxidation mediated by free radicals may be one mechanism of the reperfusion injury in the ischemic kidney (2). In vitro models of renal function have used renal slices, renal tubules, renal cells in culture, and isolated perfused kidney $(3,4)$. The use of renal slices in the evaluation of renal cellular injury is convenient because the slices are casy to prepare and the equipment is inexpensive. Many samples from the same animal can be studied at the same time under different experimental conditions, and cellular phenomena seen in vitro with renal slices are probably representative of in vivo renal function $(5-7)$. In the present study, our aim was to examine whether changes in the lipid peroxide level and various cellular functions occur during in vitro oxygenation of slices prepared from kidneys made ischemic.

Male Sprague-Dawley rats weighing $300-350 \mathrm{~g}$ were used. The animals were housed in a room with controlled temperature, humidity, and light (12-hr light-dark cycle) and provided with food and water ad libitum. Renal ischemia was induced in rats anesthetized with

\footnotetext{
*To whom all correspondence should be addressed.
}

sodium pentobarbital $(50 \mathrm{mg} / \mathrm{kg}$, i.p.). The abdomen was opened and the renal arteries of both kidneys were ligated with silk thread. The right kidney was removed as a control immediately after the ligation. The left kideny was removed after being made ischemic for 20 min. Both kidneys were placed in ice-cold $0.9 \% \mathrm{NaCl}$ for the preparation of slices from their cortices with a razor blade as described elsewhere (8). The slices were incubated at $37^{\circ} \mathrm{C}$ in a medium containing $134 \mathrm{mM}$ $\mathrm{NaCl}, 5.9 \mathrm{mM} \mathrm{KCl}, 1.5 \mathrm{mM} \mathrm{CaCl}, 1.2 \mathrm{mM} \mathrm{MgCl}_{2}, 5$ $\mathrm{mM}$ pyruvate, and $5.8 \mathrm{mM} \mathrm{N}$-2-hydroxyethylpiperazine$N$ '-2-ethanesulfonic acid titrated with $\mathrm{NaOH}$ to $\mathrm{pH} 7.4$, with $100 \%$ oxygen. After incubation, the medium was analyzed spectrophotometrically for lipid peroxides and glucose for the assay of gluconeogenesis, for which the slices were prepared from rats starved for about $24 \mathrm{hr}$ for the convenience of assaying the capacity for gluconcogenesis because this increased the capacity for gluconeogenesis from pyruvate without affecting lipid peroxidation in our preliminary experiments. Lipid peroxidation was monitored by measurement of malondialdehyde production by the thiobarbituric acid assay of Buege and Aust (9). Glucose was measured with a commercial kit (Wako Pure Chemical Industries, Ltd., Osaka). Before and after incubation, the ATP level and the level of glutathione (GSH) as a nonprotein sulfhydryl in the slices were assayed by the methods of Kimmich et al. (10) and Ellman (11), respectively. $N, N^{\prime}$-Diphenyl-p-phenylenediamine (DPPD), a strong 
antioxidant $(12,13)$, was purchased from Tokyo Kasei Kogyo Co., Ltd. (Tokyo, Japan), and dissolved in ethyl alcohol, which was added to the medium to the final concentration of $2 \%$. Other chemicals were of the highest purity available from commercial sources. Data are expressed as means $\pm \mathrm{S}$.E. Statistical analysis was done by Student's $t$-test.

Figure 1 shows the effect of renal ischemia and DPPD on lipid peroxidation (A) and ATP levels (B) in kidney cortical slices. Oxygenation of the slices prepared from ischemic kidneys significantly increased the level of lipid peroxidation. Before incubation, the slices prepared from ischemic kidneys had a lower level of ATP. ATP increased with postischemic oxygenation not only in the control slices (to $161 \%$ of the level before incubation) but also in the slices from ischemic kidneys (to 227\%), although the ATP level in the latter was still lower than that in the former after incubation. DPPD completely abolished lipid peroxidation caused by oxygenation of the slices prepared from ischemic kidney although DPPD alone reduced the level of lipid peroxides in the control slices. DPPD had no effect on ATP levels in the incubated slices.

In the next experiments, we studied the effect of ischemia and DPPD on GSH and gluconeogenesis in the slices (Fig. 2). Slices were incubated for $30 \mathrm{~min}$ because, in preliminary experiments, gluconeogenesis from pyruvate in control slices reached a sufficient capacity for measurement after $30 \mathrm{~min}$ of incubation. Lipid peroxidation was significantly increased by $30 \mathrm{~min}$ of postischemic oxygenation (the slices from control kidneys, $40.9 \pm 1.6 \mathrm{nmol} / \mathrm{g}$ wet wt.; the slices from kidneys made ischemic for $20 \mathrm{~min}, 59.4 \pm 1.0 \mathrm{nmol} / \mathrm{g}$ wet wt.) Such an increase in lipid peroxidation in the slices from ischemic kidneys was prevented by DPPD (data not shown). Postischemic oxygenation significantly decreased the GSH level and the capacity for gluconeogenesis in the slices. Such decreases were not affected by DPPD.

Paller et al. (2) have reported that restoration of the oxygen supply to ischemic kidneys increases lipid peroxidation, which causes renal injury. In our study, in vitro oxygenation of slices prepared from ischemic kidneys increased lipid peroxidation, which could be blocked by the antioxidant DPPD, suggesting that some free radicals were generated in the slices during postischemic oxygenation. Renal ischemia rapidly decreases tissue ATP $(14,15)$. This was shown by the low ATP level before incubation in the slices prepared from ischemic kidneys. Incubation increased the ATP level in both the control slices and those from ischemic kidneys, indicating that the ability of the slices to synthesize ATP may not be hindered by ischemia for $20 \mathrm{~min}$. Oxygenation decreased both the GSH content and the capacity for
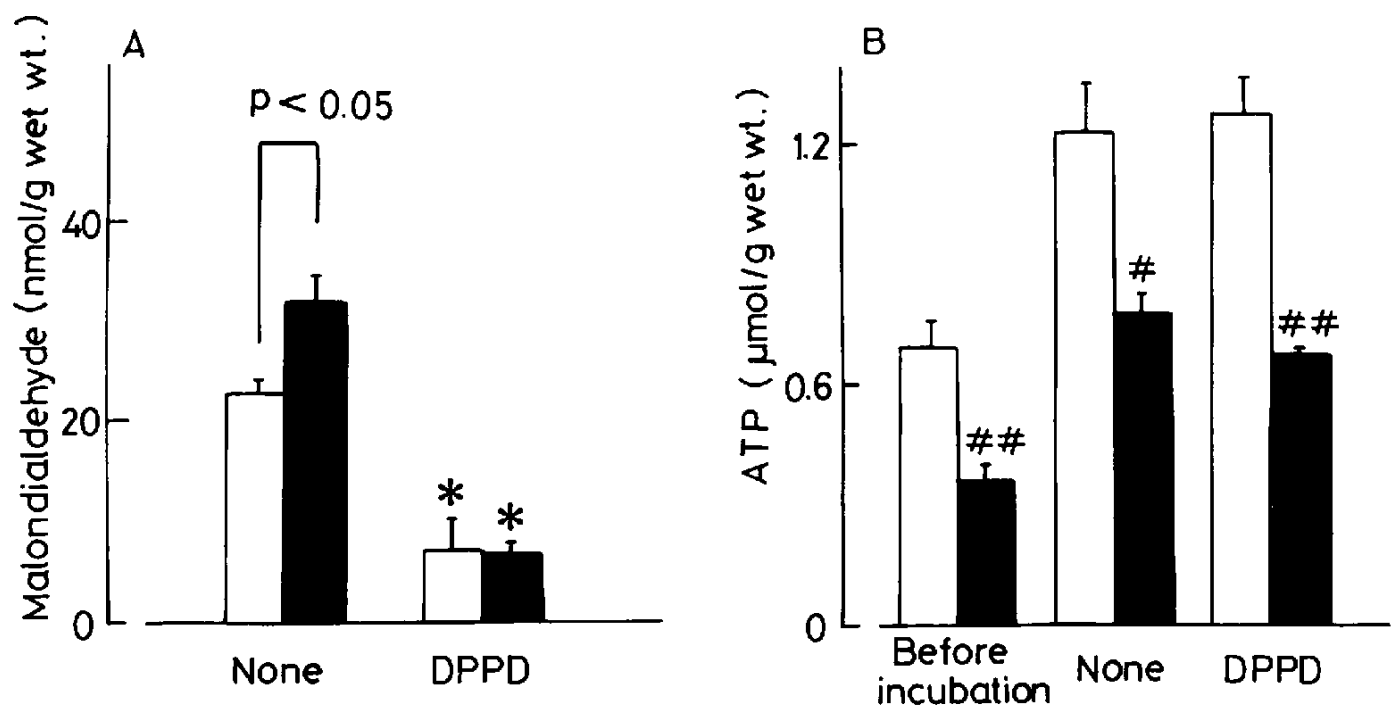

Fig. 1. Effect of ischemia and $N, N^{\prime \prime}$-diphenyl-p-phenylenediamine (DPPD) on lipid peroxidation (A) and ATP (B) levels in rat kidney cortical slices. Cortical slices prepared from control kidneys ( $\square$ ) or from kidneys made ischemic for 20 min (G) were incubated at $37^{\circ} \mathrm{C}$ for $10 \mathrm{~min}$ with or without $1 \mu \mathrm{M}$ DPPD under a $100 \%$ oxygen atmosphere. Data represent the means \pm S.E. of at least four experiments. ${ }^{*} \mathrm{P}<0.01$ compared with the corresponding "None." ${ }^{\#} \mathrm{P}<0.05$ and ${ }^{\# \#} \mathrm{P}$ $<0.01$ compared with the slices from the corresponding control kidney. 

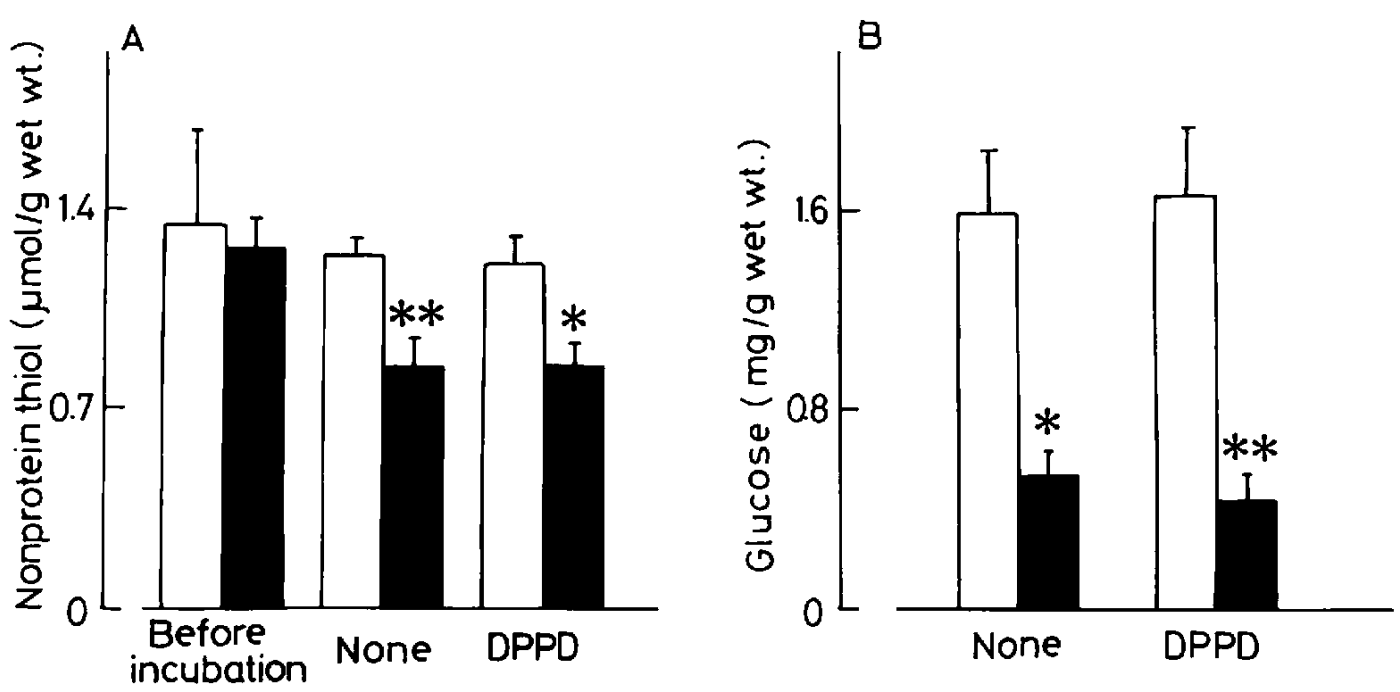

Fig. 2. Effect of ischemia and $N, N^{\prime}$-diphenyl-p-phenylenediamine (DPPD) on nonprotein thiol (A) and gluconeogenesis (B) in rat kidney cortical slices. Cortical slices prepared from control kidneys $(\square)$ or from kidneys made ischemic for 20 $\min (\square)$ were incubated at $37^{\circ} \mathrm{C}$ for $30 \mathrm{~min}$ under a $100 \%$ oxygen atmosphere. Results shown are the means $\pm \mathrm{S}$.E. of at least four experiments. ${ }^{*} \mathrm{P}<0.05$ and ${ }^{* *} \mathrm{P}<0.01$ compared with the slices from the corresponding control kidney.

gluconeogenesis in slices prepared from kidney made ischemic for $20 \mathrm{~min}$. Such decreases were not affected by DPPD. These results show that the decreased capacity for gluconeogenesis in slices from ischemic kidneys may be associated with a decrease in the GSH level during postischemic oxygenation, not with increased lipid peroxidation. Hereafter, it is necessary to consider how free radical species generated by oxygenation of slices prepared from ischemic kidneys are able to injure renal cells by decreasing the GSH level in slices.

\section{Acknowledgments \\ We thank Ms. Caroline Latta for help in preparing the manu-} script.

\section{REFERENCES}

1 McCord, J.M.: Oxygen-derived free radicals in postischemic tissue injury. N. Engl. J. Med. 312, $159-163$ (1985)

2 Paller, M.S., Hoidal, J.R. and Ferris, T.F.: Oxygen free radicals in ischemic acute renal failure in the rat. J. Clin. Invest. 74, 1156-1164 (1984)

3 Smith, M.A., Hewitt, W.R. and Hook, J.B.: In vitro methods in renal toxicology. In In Vitro Methods in Toxicology, Edited by Atterwill, C.K. and Steele, C.E., p. 13-35, Cambridge Uniyersity Press. Cambridge (1987)

4 Williams, P.D.: The application of renal cells in culture in studying drug-induced nephrotoxicity. In Vitro Cell. Dev. Biol. 25, $800-805$ (1989)

5 Berndt, W.O.: Use of the tissue slice technique for evalua- tion of renal transport processes. Environ. Health Perspect. 15, $73-88$ (1976)

6 Phelps, J.S., Gandolfi, A.J., Brendel, K. and Dorr, R.T.: Cisplatin nephrotoxicity: In vitro studies with precision-cut rabbit renal cortical slices. Toxicol. Appl. Pharmacol. 90, $501-512(1987)$

7 Smith, J.H.: The use of renal cortical slices from the Fischer 344 rat as an in vitro model to evaluate nephrotoxicity. Fundam. Appl. Toxicol, 11, 132-142 (1988)

8 Gemba, M., Tachibana, A., Sugihara, K., Hori, M. and Nakajima, M.: Inhibitory effect of lithium on $p$-aminohippurate transport in rat kidney cortex in vitro. Renal Physiol. 8. $179-188$ (1985)

9 Buege, J.A. and Aust, S.D.: Microsomal lipid peroxidation. Methods Enzymol. 52, 302-310 (1978)

10 Kimmich, G.A., Randles, J. and Brand, J.S.: Assay of picomole amounts of ATP, ADP, and AMP using the luciferase enzyme system. Anal. Biochem. 69, 187-206 (1975)

11 Ellman, G.L.: Tissue sulfhydryl groups. Arch. Biochem. Biophys. 82, $70-77$ (1959)

12 Masuda, Y. and Murano, T.: Carbon tetrachloride-induced lipid peroxidation of rat liver microsomes in vitro. Biochem. Pharmacol. 26, 2275-2282 (1977)

13 Cojocel, C., Laeschke, K.H., Inselmann, G. and Baumann, $\mathrm{K}$.: Inhibition of cephaloridine-induced lipid peroxidation. Toxicology 35, 295-305 (1985)

14 Hems, D.A. and Brosnan, J.T.: Effects of ischaemia on content of metabolites in rat liver and kidney in vivo. Biochem. J. 120, 105-111 (1970)

15 Kessler, R.H.: Effects of ischemia on the concentration of adenine nucleotides in the kidney of anesthetized dogs. Proc. Soc. Exp. Biol. Med. 134, $1091-1095$ (1970) 